\title{
EVALUATION OF VARIABILITY OF SUPRAORBITAL NOTCHES AND FORAMINA USING THREE-DIMENSIONAL COMPUTER TOMOGRAPHY VOLUME RENDERING
}

\author{
Marija Dakovic Bjelakovic ${ }^{*}$, Jelena Popovic ${ }^{2}$, Dragan Stojanov3, \\ Jelena Ignjatovic 4 , Tanja Dzopalic 5
}

\author{
${ }^{1}$ Department of Anatomy, Medical Faculty, University of Nis, Serbia \\ ${ }^{2}$ Department of Restorative Dentistry and Endodontics, Clinic of Dentistry, Medical Faculty, University of Nis, Serbia \\ ${ }^{3}$ Center of Radiology, Clinical Center of Nis, Serbia \\ 4Medical Faculty, University of Nis, Serbia \\ 5Department of Immunology, Medical Faculty, University of Nis, Serbia
}

\begin{abstract}
In this study, we aimed to estimate the anatomical variations of the supraorbital notch or supraorbital foramen $(S O N / F)$ using three-dimensional computer tomography $(3 D-C T)$ volume rendering and evaluate these variations related to gender and side.

One hundred and five adults ( 53 men and 52 women), aged 21 to 83 years, without any trauma or bone malformation of facial bones, were included in our study. Presence of $S O N / F$, its shape, diameter in millimeters, and distance from the reference point were recorded.

The most common presentation of the supraorbital passage was single SON and it was noted in $51.43 \%$ on the right and in $57.14 \%$ on the left skull side. The single SOF was noted in $3.81 \%$ on the right and $5.71 \%$ on the left skull side. Double notch was found in $1.90 \%$ on the right and in $0.95 \%$ on the left side, while double foramen was not found in subjects included in our study. SON/F was not found in $42.90 \%$ and $36.20 \%$ on the right and left side, respectively. The average SON diameter was $3.7 \pm 0.9 \mathrm{~mm}$, and the average SOF diameter was $1.7 \pm 0.5 \mathrm{~mm}$. The average distance of $S O N / F$ to the facial midline was $21.9 \pm 3.1 \mathrm{~mm}$. The average distance of $S O N / F$ to the temporal crest of the frontal bone was $26.0 \pm 3.0 \mathrm{~mm}$ and to the frontozygomatic suture was $27.5 \pm 2.6 \mathrm{~mm}$. Statistically significant difference was observed between the right and left side for the distance from $S O N / F$ to frontozygomatic suture $(p<0.01)$. No significant difference was found between the left and right sides in the other measurements $(p>0.05)$. Statistically significant differences were found between males and females for some of these parameters.

The results of this study provide useful information about the location of the supraorbital neurovascular bundle and may be used in preoperative evaluation.
\end{abstract}

Key words: Variations, supraorbital notch, supraorbital foramen, computed tomography, volume rendering

DOI: $10.21175 /$ RadProc.2016.30

\section{INTRODUCTION}

The facial foramina transmit neurovascular bundles between the cranium and facial region. They are important anatomical landmarks that need to be considered in surgery, local anesthesia and other invasive procedures in maxillofacial area. Knowledge of the regional anatomy of the face and the location of facial foramina is essential for avoiding injuries to the neurovascular bundles, which helps in decreasing morbidity and provides satisfactory results. A large variety of surgical procedures require incisions on the face and use of local anesthesia. Peripheral local anesthetic blocks of these neurovascular bundles provide excellent analgesia for the closure of simple facial wounds, biopsies, scar revisions, cosmetic procedures and during various endoscopic procedures which are increasingly being used for cosmetic facial surgery. Precise and effective analgesia can be achieved only if the surgeon is aware of the most frequent location of the exit of regional nerves. If not, injuries causing bleeding and loss of sensation in the corresponding regions of the face can occur. Excessive dissection and retraction near the neurovascular bundles can also cause scarring, leading to neuropathies and painful neuralgias [1]-[4].

The supraorbital notch or supraorbital foramen $(\mathrm{SON} / \mathrm{F})$ is a passage in the frontal bone for the supraorbital nerve, artery and veins. The human supraorbital nerve is a purely sensory nerve. It originates from fibers of the frontal nerve, which is the largest branch of the ophthalmic division of the trigeminal nerve. The supraorbital nerve traverses supraorbital notch or foramen (SON/F) and gives off nerve fibers to the vertex, thus, providing sensory innervation to the upper eyelid and conjunctiva, the lateral portion of the forehead, and the skin of the anterior scalp nearly as far back as the lambdoid suture. The supraorbital artery, a branch of ophthalmic artery, leaves the orbit through the SON/F, dividing

marijadb968@gmail.com 
into superficial and deep branches to supply the skin and muscles of the upper eyelid, forehead and scalp. According to standard descriptions in anatomy textbooks, the supraorbital notch/foramen is situated at the junction of lateral two-thirds and medial third of the supraorbital margin [5], [6]. However, most of the published studies reported that the position and the morphometric characteristics of $\mathrm{SON} / \mathrm{F}$ are not constant. In some skulls, cases of incomplete foramina, double foramina, double notch or the absence of all of them have been reported [1]-[3], [7]-[10].

Medical progress requires more precise knowledge of morphological variability of humans in order to improve diagnostic and therapeutic activity. The rapid development of Computed Tomography (CT) has resulted in new CT applications. One of these applications, volume rendering, represents a key technique for visualization of three-dimensional (3D) dataset. Its task is to display volumetric data as a relevant two-dimensional (2D) image which users can interpret. A CT volume rendering enables rendering of the CT medical data. This technique allows the exploration of fine anatomical details that would be difficult to evaluate using only axial reconstructions. $3 \mathrm{D}-\mathrm{CT}$ with volume rendering, has become an area of great clinical and basic scientifical research for the qualitative and quantitative analysis of normal and abnormal anatomic structures. In combination with the advances in computed graphic, $3 \mathrm{D}-\mathrm{CT}$ has so far been used in several different clinical applications, such as evaluation of craniofacial anomalies, dental implants planning, fractures and pathological processes [11]-[13]. It has also been used for defining unique individual aspects of complex anatomy, planning interventions and evaluating outcomes, and for anthropometric studies. Multidetector CT (MDCT) of the head is an exquisite technique for demonstrating the cranial foramina in living individuals [14]. This technique provides great facilities to maxillofacial surgeons with its excellent resolution and volume rendered images. By continuously developing software programs uninterested parts can be removed from the images and significant anatomical structures can be examined volumetrically.

In this study, we aimed to analyze anatomical variations of the $\mathrm{SON} / \mathrm{F}$ in living subjects using $3 \mathrm{D}-\mathrm{CT}$ volume rendering, and to evaluate these variations in relation to gender and side.

\section{MATERIALS AND METHODS}

The current study included 105 adult subjects, aged between 21 and 85 years, who did not have any facial bone trauma or malformation and did not have history of prior trauma or surgery on periorbital region. Data of the subjects were collected from the patients referred to the Center of Radiology, Clinical Center of Nis, Serbia, between September and December 2015 for MDCT examination of facial bones and cranial base. The study was approved by the Ethics Committee of the Clinical Centre of Nis.

Head CT examination was performed on the CT with 16 rows of detectors (GE CT/e Dual, GE Lightspeed 16; GE Medical Systems, USA). Slice thickness was $0.625 \mathrm{~mm}$ with scanning parameters 120 $\mathrm{kVp}$ and $210 \mathrm{~mA}$, without overlapping. The heads were 128 imaged beginning superior to the vertex of the skull and extending inferiorly to below the mandible. The $3 \mathrm{D}-\mathrm{CT}$ images of the skull are obtained with a technique of lighting, removal of overlying anatomic structures and rotation. Measurement and $3 \mathrm{D}$ volume rendering were conducted on workstation Advantage Workstation 4.1 (General Electric, Waukesha, USA).

The presence of different types of supraorbital passages, the dimensions and position of SON/F were analyzed. Measurements were taken in 2D based on the consideration of their application to living subjects and the use of measuring instruments, such as the coordinate caliper. Relative position was recorded by measuring distances from SON/F to reference points. All measurements were done bilaterally, and the data were compared between two genders and two sides for each measurement. The morphometric measurements, including their description and the referent points, were (Figure 1):

(1) SON Wd: The maximum width of the SON.

(2) SOF Td: The maximum transverse diameter of the SOF.

(3) SOF Vd: Maximum vertical diameter of the SOF.

(4) SOF-SOM: If a supraorbital passage was foramen, the vertical distance from the inferior margin of the SOF to the point on the superior orbital margin (SOM), in a line parallel to the facial midline.

(5) SON/F-FM: The distance from the medial margin of the SON/F to the facial midline (FM), in a line perpendicular to the facial midline.

(6) SON/F-TCFB: The distance from the lateral margin of the SON/F to the temporal crest of the frontal bone (TCFB), in a line perpendicular to the facial midline.

(7) SON/F - FZS: The distance from lateral margin of the $\mathrm{SON} / \mathrm{F}$ to the frontozygomatic suture (FZS).

The obtained results were statistically analysed. Descriptive statistics, the mean value, standard deviation, minimum, maximum and 95\% confidence interval for each of the measurements were assessed. Student's $t$ test was performed to compare the morphometric variables in relation to gender and side and Mann-Whitney U test was when data did not have normal distribution. Statistical differences were considered when the $p$ value was less than 0.05. Statistical analysis was performed using PASW Statistics 18.0. 


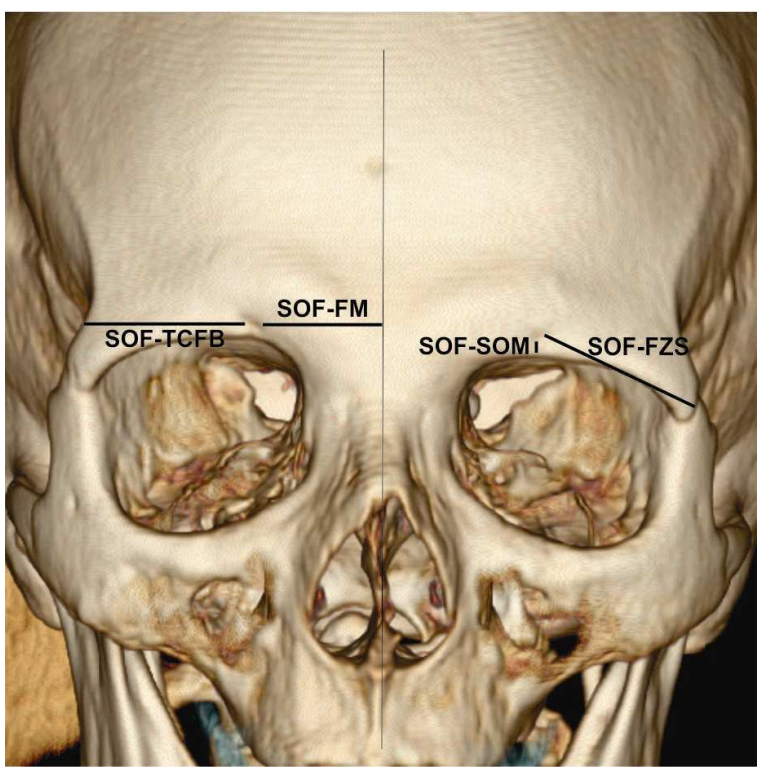

Figure 1. SON/F - supraorbital notch/foramen, SOM supraorbital margin, FM - facial midline, TCFB - temporal crest of the frontal bone, FZS - frontozygomatic suture

\section{RESULTS}

Subjects included into the study comprised 53 men (50.48\%) and 52 women (49.52\%). The mean age of subjects was \pm years (male, $66.66 \pm 10.61$; female, $60.36 \pm 16.63)$. Of the 105 crania, the supraorbital passage was present in the form of a $\mathrm{SON} / \mathrm{F}$ in 81 crania (77.14\%), unilaterally or bilaterally. The SON/F was absent in $59(56.19 \%)$ crania. Absence was noted in 35 crania (33.33\%) unilaterally and 24 (22.86\%) bilaterally. The most common presentation of the supraorbital passage was single SON and it was noted in $77(73.33 \%)$ crania. The unilateral SON has been found in 41 (39.01\%) and bilateral has been found in 36 (34.29\%) crania. The single SOF was noted in 7 (6.67\%) crania, 3 (2.86\%) unilaterally and 4 (3.81\%) bilaterally. Double SON was found only unilaterally, in 3 (2.86\%). No statistical difference was detected between the two sides. There was also no statistical difference between males and females. The data concerning the types of the supraorbital passage related to gender and side were summarized in Table 1.

Table 1. Types of the supraorbital passage related to gender and side

\begin{tabular}{|c|c|c|c|c|c|c|}
\hline \multirow[t]{2}{*}{$\mathrm{SON} / \mathrm{F}$} & \multicolumn{2}{|c|}{ Male $(n=53)$} & \multicolumn{2}{|c|}{ Female $(n=52)$} & \multicolumn{2}{|c|}{ Total $(n=105)$} \\
\hline & Right, $n(\%)$ & Left, $n(\%)$ & Right, $n(\%)$ & Left, $n(\%)$ & Right, $n(\%)$ & Left, $n(\%)$ \\
\hline Absence & 21 (20.0) & $19(18.10)$ & $24(22.90)$ & $19(18.10)$ & $45(42.90)$ & $38(36.20)$ \\
\hline Single notch & $26(24.76)$ & $30(28.57)$ & $28(26.67)$ & $30(28.57)$ & $54(51.43)$ & $60(57.14)$ \\
\hline Single foramen & $4 \quad(3.81)$ & $4 \quad(3.81)$ & - & $2(1.90)$ & $4 \quad(3.81)$ & $6 \quad(5.71)$ \\
\hline Duble notch & (1.90) & - & - & $1 \quad(0.95)$ & (1.90) & $1 \quad(0.95)$ \\
\hline
\end{tabular}

Table 2. Measurements of the supraorbital notch or foramen related to side

\begin{tabular}{|c|c|c|c|c|c|c|c|c|c|c|c|c|c|}
\hline \multirow[t]{2}{*}{ Measurement } & \multicolumn{4}{|c|}{ Total $(n=105)$} & \multicolumn{4}{|c|}{ Right $(n=105)$} & \multicolumn{4}{|c|}{ Left $(n=105)$} & \multirow[t]{2}{*}{$p$ value } \\
\hline & Mean & $\mathrm{SD}$ & Min & Max & Mean & SD & Min & Max & Mean & SD & Min & Max & \\
\hline SON Wd & 3.70 & 0.94 & 1.50 & $5 \cdot 30$ & 3.69 & 0.95 & 1.50 & 5.20 & 3.70 & 0.93 & 1.50 & $5 \cdot 30$ & 0.894 \\
\hline $\mathrm{OFF} \mathrm{Td}$ & 1.70 & 0.47 & 1.50 & 2.70 & 1.55 & 0.17 & 1.40 & 1.80 & 1.77 & 0.57 & 1.20 & 2.70 & 0.648 \\
\hline $\mathrm{Vd}$ & 1.49 & 0.47 & 0.80 & 2.50 & 1.42 & 0.35 & 1.00 & 1.80 & 1.53 & 0.55 & 0.80 & 2.50 & 0.236 \\
\hline SOF-SOM & 4.98 & 2.51 & 2.00 & 9.30 & $5 \cdot 33$ & 1.02 & 4.60 & 6.50 & 4.83 & 1.12 & 2.00 & 9.30 & 0.383 \\
\hline SON/F-FML & 21.90 & 3.10 & 16.70 & 30.5 & 21.88 & 3.13 & 16.70 & 29.30 & 21.92 & 3.11 & 16.70 & 30.50 & 0.940 \\
\hline SON/F-TCFB & 25.99 & 3.03 & 18.70 & 38.20 & 25.66 & 2.20 & 21.50 & 31.30 & 26.27 & 3.58 & 18.70 & 38.20 & $0.005 * *$ \\
\hline SON/F-FZS & 27.52 & 2.58 & 22.10 & 35.70 & 27.07 & 2.16 & 22.10 & 30.80 & 27.91 & 2.84 & 22.20 & 35.70 & 0.081 \\
\hline
\end{tabular}

All measurements are in $\mathrm{mm}$. SON - supraorbital notch; SOF - supraorbital foramen; Wd - width; Td - transversal diameter;

Vd - vertical diameter; SOM - supraorbital margin; FML - facial midline; TCFB - temporal crest of the frontal bone; FZS - frontozygomatic suture; $* \mathrm{p}<0.05 ; * * \mathrm{p}<0.01$

Table 3. Measurements of the supraorbital notch or foramen related to gender

\begin{tabular}{|l|r|r|r|r|r|r|r|r|l|}
\hline Measurement & \multicolumn{4}{|c|}{ Males $(n=53)$} & \multicolumn{3}{c|}{ Females $(n=52)$} & \multirow{2}{*}{ p value } \\
\cline { 2 - 8 } & Mean & \multicolumn{1}{c|}{ SD } & \multicolumn{1}{c|}{ Min } & \multicolumn{1}{c|}{ Max } & Mean & \multicolumn{1}{c|}{ SD } & \multicolumn{1}{c|}{ Min } & \multicolumn{1}{c|}{ Max } & \\
\hline SON Wd & 3.94 & 0.91 & 1.50 & 5.20 & 3.45 & 0.90 & 1.50 & 5.30 & $0.002^{* *}$ \\
SOF Td & 1.77 & 0.49 & 1.40 & 2.70 & 1.40 & 0.28 & 1.20 & 1.60 & 0.006 \\
SOF Vd & 1.43 & 0.35 & 1.00 & 1.80 & 1.53 & 0.55 & 0.80 & 2.50 & 0.236 \\
SOF-SOM & 5.43 & 2.61 & 2.00 & 9.30 & 3.30 & 1.56 & 2.20 & 4.40 & 0.486 \\
SON/F-FML & 22.52 & 3.21 & 16.70 & 29.30 & 21.25 & 2.87 & 16.70 & 30.50 & 0.361 \\
SON/F-TCFB & 26.32 & 2.98 & 18.70 & 33.90 & 25.64 & 3.07 & 19.50 & 38.20 & 0.229 \\
SON/F-FZS & 27.95 & 2.61 & 22.70 & 35.70 & 27.08 & 2.49 & 22.10 & 32.40 & 0.071 \\
\hline
\end{tabular}

All measurements are in mm. SON - supraorbital notch; SOF - supraorbital foramen; Wd - width; Td - transversal diameter;

Vd - vertical diameter; SOM - supraorbital margin; FML - facial midline; TCFB - temporal crest of the frontal bone; FZS - frontozygomatic suture; $* p<0.05 ; * * p<0.005$. 
In cases when the supraorbital passage was in form of a SON, it average width was $3.70 \pm 0.94 \mathrm{~mm}(95 \%$ CI: $3.52-3.88$ ). When the supraorbital passage was in the form of a SOF, it was on an average $4.98 \pm 2.51 \mathrm{~mm}$ (95\% CI: $3.19-6.78$ ) superior to the superior orbital margin. The average transversal diameter of the SOF was $1.70 \pm 0.47 \mathrm{~mm}$ (95\% CI: 1.38 - 2.01), and the average vertical diameter was $1.49 \pm 0.47 \mathrm{~mm}$ (95\% CI: $1.17-1.81$ ). The average distance of the $\mathrm{SON} / \mathrm{F}$ to the facial midline was $21.90 \pm 3.10 \mathrm{~mm}(95 \% \mathrm{CI}: 21.33$ 22.47). The average distance of the $\mathrm{SON} / \mathrm{F}$ to the temporal crest of the frontal bone was $25.99 \pm 3.03$ $\mathrm{mm}$ (95\% CI: 25.43 - 26.55). The average distance from the SON/F to the frontozygomatic suture was $27.52 \pm$ 2.58 mm (95\% CI: 27.05 - 27.99).

The comparison between the measurements of the right and left SON/F are summarized in Table 2. Statistically significant difference was observed between the right and left side for the distance from $\mathrm{SON} / \mathrm{F}$ to frontozygomatic suture $(p<0.01)$. No significant difference was found between the left and right sides in the other measurements $(p>0.05)$.

The comparison between the measurements of male and female crania is summarized in Table 3. All mean values, except the vertical diameter of the SOF, were higher in male than in female. Statistically significant difference was found between males and females for width of the SON $(p<0.01)$. There was no statistically significant difference between the male and female crania for all the other parameters $(p>0.05)$.

\section{DisCUSSION}

Anatomical variations of facial foramina are of great importance in a standard surgical practice. The evaluation of localization of these anatomical landmarks enables determination of the position of neurovascular bundles that pass through the corresponding openings with the aim of increasing successful interventions and avoiding sequel. Knowledge of the anatomical characteristics of the supraorbital structures is crucial for identifying the danger zone in trauma treatment (e.g. brow and forehead wounds, trochlear trauma, foreign bodies in the brow region, marginal orbital fractures, supraorbital neuroma), in surgery (e.g. anterior orbital approach, fronto-glabellar reconstruction flap, blepharospasm surgery, Graves disease surgery, ptosis surgery) as well as in anesthesiology (e.g. superior orbital injection, peribulbar injection) when regional nerve blockage is planned, and in the treatment of migrene [10], [15]-[18]. Facial aesthetic surgery relies on the knowledge of regional anatomy in order to avoid injuries of neurovascular structures and increase successful interventions. Open and endoscopic forehead and brow lift techniques have become a standard procedure in esthetic surgery, even though there is a risk of a potential injury of the neurovascular bundle if the exit point and the branching pattern of the supraorbital nerve and vessels are unknown. Traditionally, a forehead flap is designed in the orbitofrontal region based on the dual blood supply provided by the right and left supraorbital arteries. Injuries of supraorbital neurovascular structures can lead to temporary or permanent loss of sensation in the nerve innervation region and partial flap necrosis [19]-
[21]. Excessive dissection and retraction close to such neurovascular bundles can cause scarring whereas compression of the nerve by scar tissue or a connective tissue band, intraneural local anesthetics or nerve retraction during surgery may cause supraorbital neuralgia. Diagnostic and therapeutic nerve blocks are used in hemifacial neuralgia and have been systematically performed on various types of headache [22]-[24].

Various anatomical studies have been conducted with the aim to describe anatomical variations of $\mathrm{SON} / \mathrm{F}$ and to examine the exit points of the supraorbital nerve and vessels from the orbit. The frequency of occurrence of SON and SOF varies in different population. The findings of the previous studies have shown the higher incidence of the SON than SOF, ranging from 47.50 to $92.5 \%$ [1], [7], [9], [10], [26]. The frequency of SOF ranged from $8 \%$ to $51 \%$ depending upon the studied population [25]. In our study, SON was the most frequent presentation of the supraorbital passage as well. It was noted in $73.33 \%$ of the subjects, $51.43 \%$ in the right and $57.14 \%$ in the left side. The SOF was present in $6.67 \%$ of the subjects, $3.81 \%$ in the right and $5.71 \%$ in the left side. The shape of human cranium and distribution of the supraorbital structures may be influenced by numerous factors, both genetic and environmental, and also by the climate [27], [28]. Tomaszewska et al. [28] supposed that the type and frequency of the supraorbital structures in modern human population may depend on the climatic conditions in which the population lived. Analyzing 1978 orbits from the skulls collected in three climatic regions, warm, temperate and cold, these authors discovered that the frequency of SON was the highest in the sample from warm climates (54.5\%), and the lowest in the sample from cold climatic conditions (44.0\%). In contrast, the highest frequency of SOF was in the skull from cold climatic conditions (35.4\%). In samples from warm climates, the frequency of SOF was the lowest $(16.4 \%)$. In the same study, a smooth supraorbital rim with absent SON/F was in $16.8 \%$ of all the studied orbits. In the samples from a temperate climate, a smooth supraorbital rim was most frequent (20.8\%), and in the samples from a cold climate, the frequency of the smooth supraorbital rim was the lowest (11.3\%). Agthong et al. [2] stated that the absence of SON/F was $5.5 \%$ in the right and $10.0 \%$ in the left side. In our study, a smooth supraorbital rim with the absence of SON/F was reported in $56.19 \%$ of subjects, in $42.90 \%$ in the right and $36.20 \%$ in the left side. Compared to the previous studies, the difference in our findings might be due to racial difference of the studied populations. The shape and dimensions of the supraorbital passage may affect the fixation of the neurovascular bundle. The presence of the SOF implies that the supraorbital neurovascular bundle is relatively fixed in position and therefore at a greater risk during surgical dissection, as during retraction it is more likely to be stretched. Therefore extra care has to be exercised during reflection of scalp flaps [29]. The results of the study conducted on 79 dry adult human skulls showed that there was wide variation in the width of the $\mathrm{SON} / \mathrm{F}$, ranging from $1.6 \mathrm{~mm}$ to $10.4 \mathrm{~mm}$ [3].

The $\mathrm{SON} / \mathrm{F}$ is identified by palpation of the supraorbital margin of the frontal bony. However, palpating the SON/F alone is not sufficient in locating the supraorbital neurovascular bundle in all cases. The 
supraorbital neurovascular bundle might exit through SOF or SON as well as below the smooth supraorbital margin. When the SON/F is not palpable the position of the supraorbital neurovascular bundle can be determined according to other anatomical landmarks. Knowing the relative distance of the supraorbital passage from the midline, temporal crest of the frontal bone and the frontozygomatic suture may help in defining the safe zone during surgical procedures of the supraorbital region. Our results showed that the mean distance from the SON/F to the facial midline were $21.88 \pm 3.13$ and $21.92 \pm 3.11 \mathrm{~mm}$ in the right and left side, and $22.52 \pm 3.21$ and $21.25 \pm 2.87 \mathrm{~mm}$ in male and female, respectively. There was no statistically significant difference between sides and gender. The results of the previous studies conducted on the macerated and cadaveric skulls showed that the mean distance of the SON/F from the midline ranges from $20 \mathrm{~mm}$ to $30 \mathrm{~mm}$ [2], [10], [29], and from $22 \mathrm{~mm}$ to 31 $\mathrm{mm}$ [1], [8], [26], [29]. Gupta [3] reported that the mean distance of SON/F from the facial midline was about $24 \mathrm{~mm}$ on both sides. Although the range was from $16 \mathrm{~mm}$ to $39 \mathrm{~mm}$, in $93.7 \%$ of the skulls, the values were between 20 and $30 \mathrm{~mm}$. Previous studies have shown poor symmetry between sides [1], [7], [10]. The wide variation in the $\mathrm{SON} / \mathrm{F}$ midline distance indicates that the initial anesthetic injection should be given around the palpable $\mathrm{SON}, 20-50 \mathrm{~mm}$ from the midline. An additional injection at more lateral points, 30 to $50 \mathrm{~mm}$ from the facial midline, may help in getting a complete nerve blockage if the initial attempt was unsuccessful. Finally, complete forehead anesthesia seems appropriate in connection with surgical procedures along the supraorbital margin of 15 and at least $50 \mathrm{~mm}$ from the midline [17]. Although some authors used midline of the scull as landmark in analyzing of facial foramina, the intraoperative identification of this line could be difficult [3], [10]. The temporal crest of the frontal bone and frontozygomatic suture may be better landmarks for intraoperative localization of the supraorbital nerve and vessels [1] and therapeutic blockade of supraorbital nerve in migraine patients [4]. Our results showed that the mean distance from the $\mathrm{SON} / \mathrm{F}$ to the temporal crest was $25.66 \pm 2.20$ and $26.27 \pm 3.58 \mathrm{~mm}$ in the right and left side, and $26.32 \pm$ 2.98 and $25.64 \pm 3.07 \mathrm{~mm}$ in men and women, respectively. There was statistically significant difference ( $p<0.01$ ) between the right and left side, but there was no statistically significant difference between men and women. Chrcanovic et al. [10] reported that the mean value of the distance was 24.32 in the right and 22.81 $\mathrm{mm}$ in left side, which is a statistically significant difference between sides and is also compatible to the results in our study.

The supraorbital region of living subjects was analyzed in our study. We compared our findings to those of the previous studies conducted on macerated and cadaveric skulls. However, such comparison is limited, considering different methodological approach. As far as we know, there's only one similar study conducted on living subjects. Turhan-Haktanir et al. [30] examined variations of $\mathrm{SON} / \mathrm{F}$ on 399 living subjects using MDCT and evaluated the obtained results according to gender and side. Their results showed that the most common presentation of the supraorbital passage was a single SON $(69.4 \%$ in the right and $68.2 \%$ in the left) followed by a single foramen (12.4\% in the right and $14.5 \%$ in the left). The absence of SON/F was reported in $11.5 \%$ in the right and $12.5 \%$ in the left side. The diameter of the SON was $3.5 \pm 1.4 \mathrm{~mm}$, and SOF was $2.3 \pm 0.6 \mathrm{~mm}$. The diameters of SON were significantly higher in males than females, whereas those of the SOF did not show any significant differences. Distances from SON/F to facial midline were 32.3 and $25.8 \mathrm{~mm}$ in the right, and 29.1 and $24.6 \mathrm{~mm}$ in left sides, in men and women, respectively. The correct data comparison can be made only with a study with the same research topic and the same methodology. We compared the results of our CT measurements with the results of the previous studies conducted on living subjects as well. We concluded that our findings are generally similar to the previous reports. However, our study is more comprehensive since we used more parameters for the evaluation of the anatomical characteristics of the supraorbital passage. We determined the position of the supraorbital passage according to the facial midline and the temporal crest of the frontal bone and frontozygomatic suture, which is not analyzed in the previous study. The data could be useful to surgeons considering that following anatomical landmarks and extreme caution in surgical interventions may lead to the prevention of iatrogenic injuries of the supraorbital neurovascular bundle.

The definitive bony shapes are influenced by developing and ossify processes. The frontal bone is developed in membrane, in which two primary centers appear in the eight week, one above each supraorbital margin. The secondary centers appear later. At birth the bone consists of two pieces, separated by the frontal suture. These separated halves come into contact in the first year, fuse in the middle region of the frontal suture in second year, and are completely joined by the seven or eight year [5], [31]. In $25 \%$ of individuals, the SON is converted into a SOF by ossification of the periosteal supraorbital ligament closing it [7]. In a comparative study of fetal and adult human skulls, Londhe et al. [32] showed that complete supraorbital foramina were not seen in any of the fetal skulls, but in adult skulls bilateral foramina occurred in $13.86 \%$. The absence of all the features (SON, SOF and incomplete SOF) was not seen in any adult skulls, but was observed in $6.77 \%$ of fetal skulls. Multiple facial foramina have been associated with the branching of blood vessels and nerves during development. The distribution of the supraorbital structures and their differences which exist between adult forms may be influenced by genetic, hormonal and environmental factors during development and growth of skull [5], [25], [28], [31].

\section{CONCLUSION}

The supraorbital passage shows variations in shape, size and position. SON was observed more often than SOF, though there are numerous variations of the exit point of the supraorbital nerve and vessels. The position of SON/F is not constant in every individual.

Acknowledgement. Contract grant sponsor: Ministry of Science and Technological Development of Republic of Serbia; contract grant number: 175092. 


\section{REFERENCES}

1. B. Cutright, N. Quillopa and W. Schubert, "An Antropometric Analysis of the Key Foramina for Maxillofacial Surgery," J. Oral. Maxillofac. Surg, vol. 61, no. 3, pp. 354-357, Mar. 2003

2. S. Agthong, T. Huanmanop and V. Chentanez, "Anatomical Variations of the Supraorbital, Infraorbital and Mental Foramina Related to the Gender and Side," J. Oral. Maxillofac. Surg, vol. 63, no. 6, pp. 800-804, June 2005

3. T. Gupta, "Localization of the Important Facial Foramina Encountered in Maxillo-Facial Surgery," Clin. Anat, vol. 21, no. 7, pp. 633-644, Oct. 2008

4. C.A. Caputi and V. Firetto, "Therapeutic Blockade of Greater Occipital and Supraorbital Nerves in Migraine Patients," Headache, vol. 37, no. 3, pp. 174-179, Mar. 1997

5. Gray's Anatomy, 37th ed., P.L. Williams, R. Warwick, M. Dyson and L.H. Bannister, Eds., Edinburg, UK: Churchill and Livingstone, 1989

6. K.L. Moore and A.F. Dalley, Clinically Oriented Anatomy, 5th ed., Philadelphia (PA), USA: Lippincott Williams \& Wilkins, 2006

7. R.C. Webster, J.M. Gaunt, U.S. Hamdan, N.S. Fuleihan, P.R. Giandello and R.C. Smith, "Supraorbital and Supratrochlear Notches and Foramina: Anatomical Variations and Surgical Relevance," Laryngoscope, vol. 96, no. 3, pp. 311-315, Mar. 1986

8. G.M. Beer, R. Putz, K. Mager, M. Schumacher and W. Keil, "Variations of the Fronatal Exit of the Supraorbital Nerve: An Anatomic Study," Plast. Reconstr. Surg., vol. 102, no. 2, pp. 334-341, Aug. 1998

9. C. Saylam, M.A. Ozer, C. Ozek and T. Gurler, "Anatomical Variations of the Frontal and Supraorbital Transcranial Passages", J. Craniofac. Surg, vol. 14, no. 1, pp. 10-12, Jan. 2003

10. B.R. Chrcanovic, M.H.N.G. Abreu and A.L.N. Custodio, "A Morphometric Analysis of the Supraorbital and Infraorbital Foramina Relative to Surgical Landmarcs," Surg. Radiol. Anat, vol. 33, no. 4, pp. 329-335, May 2011

11. M.G.P. Cavalcanti, S.S. Rocha and M.W. Vannier, "Craniofacial Measurements Based on 3D-CT Volume Rendering: Implications for Clinical Applications," Dentomaxillofac. Radiol., vol. 33, no. 3, pp. 170-176, May 2004

12. M.G.P. Cavalcant, A. Ruprecht and M.W. Vannier, " $3 \mathrm{D}$ Volume Rendering Using Multislice CT for Dental Implants,” Dentomaxillofac. Radiol., vol. 31, no.4, pp. 218-223, Jul. 2002

13. T. Rodt et al., "Evaluation of Surface and Volume Rendering in 3D-CT of Facial Fractures," Dentomaxillofac. Radiol., vol. 35, no. 4, pp. 227-231, Jul. 2006

14. J.K. Berge and R.A. Bergman, "Variations in Size and in Symmetry of Foramina of the Human Skull," Clin. Anat., vol. 14, no. 6, pp. 406-413, Nov. 2001

15. M. Levin, "Nerve blocks in the Treatment of Headache," Neurotherapeutics, vol. 7, no. 2, pp. 197-203, Apr. 2010

16. R.W. Evans and J.A. Pareja, "Supraorbital Neuralgia," Headache, vol. 49, no. 2, pp. 278-281, Feb. 2009

17. S. Erdogmus and F. Govsa, "Anatomy of the Supraorbital Region and the Evaluation of it for the Reconstruction of Facial Defects," J. Craniofac. Surg, vol. 18, no. 1, pp. 104-112, Jan. 2007
18. A.L. Cuzalina and J.D. Holmes, "A Simple and Reliable Landmark for Identification of the Supraorbital Nerve in Surgery of the Forehead: An In Vivo Anatomical Study," J. Oral. Maxillofac. Surg, vol. 63, no. 1, pp. 25-27, Jan. 2005

19. D. Benvenuti, "Endoscopic Brow Lifts with Injury to Supraorbital Nerve and Neuroma Formation," Plast. Reconstr. Surg., vol. 104, no. 1, pp. 297-298, Jul. 1999

20. M.F. Fatah, "Innervation and Functional Reconstruction of the Forehead," Int. J. Plast. Surg., vol. 44, no. 5, pp. 351-358, Jul. 1991

21. L.M. Di Francesco, M.A. Codner and C.D. McCord, "Upper Eyelid Reconstruction," Plast. Reconstr. Surg., vol. 114, no. 7, pp. 98e-107e, Dec. 2004

22. R. Watson and K. Leslie, "Nerve Blocks versus Subcutaneous Infiltration for Stereotactic Frame Placement," Anesth. Analg., vol. 92, no. 2, pp. 424-427, Feb. 2001

23. D.S. Klein and R.E. Schmid Jr., "Chronic Headache Resuting from Postoperative Supraorbital Neuralgia," Anest. Analg., vol. 73, no. 4, pp. 490-491, Oct. 1991

24. O. Sjaastad, A. Stolt-Nielsen, J.A. Pareja, T.A. Fredriksen and M. Vincent, "Supraorbital Neuralgia: On the Clinical Manifestations and a Possible Therapeutic Approach," Headache, vol. 39, no. 3, pp. 204-212, Mar. 1999

25. A.C. Berry "Factors Affecting the Incidence of NonMetrical Skeletal Variants," J. Anat., vol. 120, Pt 3, pp. 519-535, Dec. 1975

26. M.S. Chung, H.J. Kim, H.S. Kang and I.H. Chung, "Locational Relationship of the Supraorbital Notch or Foramen Intraorbital and Mental Foramina in Koreans," Acta Anat., vol. 154, no. 2, pp. 162-166, 1995

27. K. Harvati and T.D. Weaver, "Human Cranial Anatomy and the Differential Preservation of Population History and Climate Signatures," Anat. Rec. A, vol. 288, no. 12, pp. 1225-1233, Dec. 2006

28. A. Tomaszewska, J. Tomczyk and B. Kwiatkowska, "Characterisation of the Supraorbital Foramen and Notch as an Exit Route for the Supraorbital Nerve in Populations from Different Climatic Conditions," Homo, vol. 64 , no. 1, pp. 58-70, Feb. 2013

29. A.C. Cheng, H.K. Yuen, P.W. Lucas, D.S. Lam and K.F. So, "Characterization and Localization of the Supraorbital and Frontal Exits of the Supraorbital Nerve in Chinese: An Anatomic Study," Ophthal. Plast. Reconstr. Surg, vol. 22, no. 3, pp. 209-213, May-June 2006

30. N. Turhan-Haktanir, A. Aycicek, A. Haktanir and Y. Demir, "Variations of Supraorbital Foramina in Living Subjects Evaluated with Multidetector Computed Tomography," Head Neck, vol. 30, no. 9, pp. 1211-1215, Sep. 2008

31. V.T. Inman, J.B. de C. M. Saunders, "The Ossification of the Human Frontal Bone: With Special Reference to its Presumed Pre- and Post-Frontal Elements," J. Anat., vol. 71, Pt 3, pp. 383-394, Apr. 1937

32. S.R. Londhe, A.S. Jadhav, A.G. Gosavi, "Study of the Supra Orbital Notch and Foramen in Adult and Foetal Human Skulls," Al Ameen J. Med. Sci., vol. 4, no. 4, pp. 386-390, 2011 